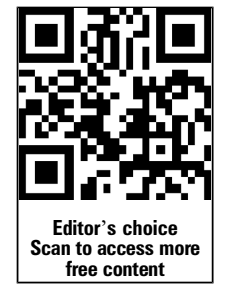

Department of Pathology and Laboratory Medicine, University of Pennsylvania, Philadelphia, Pennsylvania, USA

\section{Correspondence to}

Dr M Carolina Reyes,

Department of Pathology and Laboratory Medicine, University of Pennsylvania, 3400 Spruce Street, 2Donner, Philadelphia, PA 19104, USA;

Carolina.Reyes@uphs.upenn.edu

Received 4 December 2013 Accepted 9 December 2013 Published Online First

7 January 2014

\title{
An update on vulvar intraepithelial neoplasia: terminology and a practical approach to diagnosis
}

\author{
M Carolina Reyes, Kumarasen Cooper
}

\section{ABSTRACT}

There are two distinct types of vulvar intraepithelial neoplasia (VIN), which differ in their clinical presentation, aetiology, pathogenesis and histological/ immunophenotypical features. One form driven by highrisk human papilloma virus infection usually occurs in young women and has been termed classic or usual VIN (UVIN). The other, not related to viral infection, occurs in postmenopausal women with chronic skin conditions such as lichen sclerosus and lichen simplex chronicus and is termed differentiated or simplex-type VIN. The latter is the precursor lesion of the most common type of squamous cell carcinoma (SCC) in the vulva, namely keratinizing SCC (representing $60 \%$ of cases). In contrast, uVIN usually gives rise to basaloid or warty SCC $(40 \%$ of cases). The histological features of uVIN are similar to those of high grade lesions encountered in other lower anogenital tract sites (hyperchomatic nuclei with high nuclear to cytoplasmic ratios and increased mitotic activity). However, differentiated VIN has very subtle histopathological changes and often escapes diagnosis. Since UVIN is driven by high-risk human papilloma virus infections, p16 immunohistochemistry is diffusely positive in these lesions and is characterized with a high Ki-67 proliferation index. In contrast, differentiated or simplex-type VIN is consistently negative for p16 and the majority of the cases harbour TP53 mutations, correlating with p53 positivity by immunohistochemistry.

\section{INTRODUCTION}

Vulvar squamous cell carcinoma (SCC) is an uncommon malignant neoplasm which represents approximately $4 \%$ of all genital cancers in women, but accounts for $>90 \%$ of vulvar malignant tumours. ${ }^{1}$ Two-thirds of cases occur in women older than 60 years. There are two distinct types of vulvar intraepithelial neoplasia (VIN), which differ from each other in terms of aetiology, pathogenesis and clinical significance. One form is associated with high-risk human papilloma virus (HPV) infection, which gives rise to basaloid or warty SCCs and the second is associated with chronic inflammatory skin conditions, independent of HPV infection, often driven by p53 mutations and a precursor of keratinizing SCCs. ${ }^{2-5}$

In 2004 the International Society for the Study of Vulvar Disease (ISSVD) introduced the current two-tier classification for VIN. ${ }^{6}$ On one hand is the HPV-associated classic or usual VIN (uVIN), which encompasses high-grade lesions (VIN 2-3). The classification did not include grading of VIN and lesions formerly called VIN1 were placed in the condyloma acuminata category. The second VIN type is the HPV-independent differentiated or simplex-type VIN (dVIN). ${ }^{6}$ dVIN is considered to be a high-grade lesion, and therefore is not graded.

\section{USUAL VULVAR INTRAEPITHELIAL NEOPLASIA (CLASSIC VIN, UVIN)}

Basaloid/warty SCCs develop from classic or usual VIN (uVIN) which occurs more commonly, but not solely, in relatively young women between the ages of 40 years and 50 years and is associated with high-risk HPV infection, most often HPV 16 and less commonly HPV 18 or HPV $33 .^{7}$ Therefore, risk factors are similar to those of cervical neoplasia related to acquisition of HPV infection, namely multiple sexual partners, impaired immunological status, smoking habits and young age of first intercourse. $^{3}{ }^{8-10}$ In addition, uVIN is usually multifocal, multicentric and therefore associated with other lower anogenital intraepithelial neoplasia including cervical, vaginal and anal.

There has been an increase in the incidence of uVIN and in some countries the incidence has doubled in the past 10 years. ${ }^{10} 11$

\section{Gross findings}

Low-grade uVIN presents usually as single or multiple pale-whitish areas, whereas high-grade uVIN presents as multifocal raised plaques or papules that tend to coalesce. A small percentage of the lesions (10\%) may be hyperpigmented. There is a high frequency of mutlifocality in patients presenting with multiple lesions within the lower female anogenital tract. ${ }^{12}$

\section{Microscopic findings}

Histological changes seen in uVIN are associated with integration of high-risk oncogenic HPV infection into the host genome. The epidermis is usually thickened with parakeratosis and hyperkeratosis. At low power, loss of cell maturation is appreciated with nuclear hyperchromasia, increased mitotic figures, pleomorphism and high nuclear to cytoplasmic ratios. ${ }^{4}$ uVIN has been divided into basaloid (undifferentiated) and warty (condylomatous) subtypes. ${ }^{4}$ Basaloid VIN typically presents as a flat lesion composed of small, uniform cells resembling basal cells with high nuclear to cytoplasmic ratios and minimal koilocytic changes replacing the whole thickness of the epidermis (figure 1A). On the other hand warty VIN shows acanthosis, with wide and deep rete ridges and prominent koilocytic changes (figure 1B). However, there is frequent overlap between the two patterns with some VIN cases showing warty and basaloid features, suggesting they are the spectrum of a single disease ${ }^{25}$ without the need to divide classic VIN into subtypes.

Depending on the proportion of immature cells in the epithelium and similar to cervical lesions, in 
A

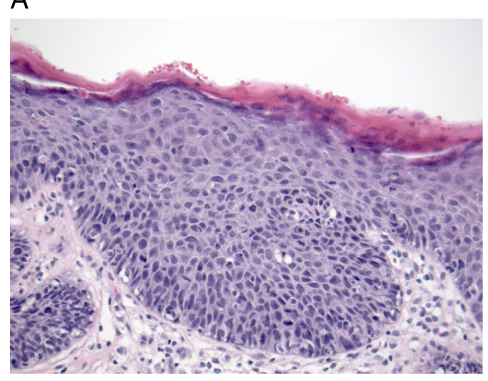

B

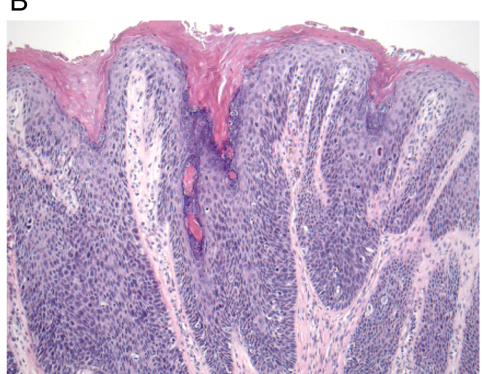

C

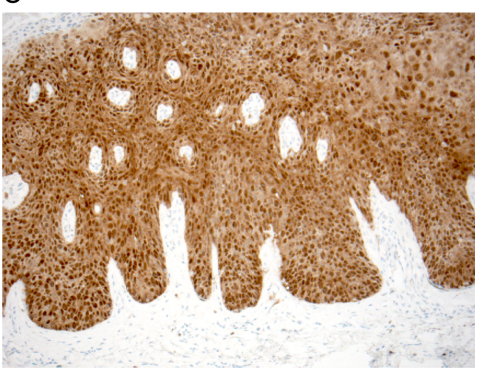

Figure 1 Vulvar intraepithelial neoplasia of the usual type (uVIN). (A) Basaloid subtype, undifferentiated cells with high nuclear to cytoplasmic ratios replace whole thickness epithelium. (B) Warty uVIN showing acanthosis, wide and deep rete ridges with koilocytic changes. (C) Diffuse, band-like p16 staining.

the current WHO grading system uVIN is divided into three grades (uVIN1-3). However, in contrast to low-grade cervical lesions (cervical intraepithelial neoplasia 1), VIN1 lesions are extremely rare and most lesions in the vulva with koilocytic changes and low-grade dysplasia represent condyloma acuminata driven by low-risk HPV types 6 and $11,{ }^{13}{ }^{14}$ which do not progress to invasive SCC. For this reason, the ISSVD revised the VIN classification in $2004^{6} 15$ and proposed abandonment of the VIN1 category. Also, since there is poor diagnostic reproducibility among pathologists with high-grade lesions such as VIN2 and VIN3, the ISSVD proposed these two categories to be combined into a single category termed classic or uVIN.

With immunohistochemistry, typically uVIN lesions show strong nuclear, cytoplasmic band-like pattern of staining with p16 (figure 1C) and increased proliferation activity with Ki-67 where positive cells extend into the upper two-thirds of the epithelial thickness.

\section{Differential diagnosis}

The differential diagnosis of uVIN includes benign processes such as reactive epithelial changes and malignant ones including vulvar Paget's disease and malignant melanoma. Whenever one is considering a reactive process p16 immunostain will help in the differential diagnosis. In reactive/benign processes $\mathrm{p} 16$ will be negative or focally weakly positive. In contrast, p16 in uVIN will show diffuse, strong, band-like labelling. ${ }^{16}$

Vulvar Paget's disease is characterized by large tumour cells with pale to eosinophilic cytoplasm within the epidermis. By immunohistochemistry these cells are positive for CK7, CEA, CAM 5.2 and GCDFP-15. ${ }^{17}$ These markers are negative in uVIN. ${ }^{18}$ Malignant melanoma will be positive for melanocytic markers such as HMB-45, S100 and Melan-A.

\section{Natural history}

Because uVIN usually presents as a clinically visible lesion, most uVIN cases will be identified by the gynaecologist during colposcopic examination. Hence, excisional biopsy will result in a low progression to SCC (3\%). ${ }^{19}$ Untreated, progression of uVIN to carcinoma is still low, varying between $10-12 \% .^{20}{ }^{21}$ The risk of progression to invasive cancer is lower in patients younger than 35 years $^{22}$ whereas in women older than 45 years of age or immunosuppressed, the risk of progression is higher. ${ }^{20}$

\section{DIFFERENTIATED OR SIMPLEX-TYPE VULVAR INTRAEPITHELIAL NEOPLASIA}

Invasive keratinizing SCC typically develops from an in situ lesion termed differentiated VIN (dVIN) or simplex-type VIN. Although dVIN can occur in young patients, this type of VIN is usually found in postmenopausal women with a mean age of 68 years $^{23}$ and tends to be unifocal and unicentric. ${ }^{24}$ Frequently dVIN develops in women with chronic dermatological diseases such as squamous cell hyperplasia, lichen sclerosus (LS) and lichen simplex chronicus. ${ }^{2} 25$ In addition, mutation of the p53 gene seems to be an early event in the development of $\mathrm{dVIN}^{26}$ with studies showing identical p53 mutations in LS and adjacent SCC. $^{27}$

\section{Gross findings}

dVIN is found in patients with chronic skin conditions related to LS, squamous cell hyperplasia and lichen simplex chronicus. However, clinical presentation is non-specific with patients often being asymptomatic. They may present with focal discolouration, ill-defined white plaques as well as red hyperkeratotic lesions. $^{2}$ Pruritus and pain have been observed in up to $60 \%$ of women. ${ }^{28}$

\section{Microscopic findings}

Histological findings of dVIN are subtle, not easily recognized by general pathologists ${ }^{29}$ and therefore often misdiagnosed as a benign dermatosis. ${ }^{23}$ dVIN is characterized by a thickened parakeratotic epithelium with elongation and anastomosing rete ridges (figure 2A). ${ }^{5}$ Marked cellular atypia is confined to the basal and parabasal cells of the epidermis comprising abnormal squamous cells with atypical mitosis in the basal layer (figure 2B). The upper epithelium is highly differentiated with epithelial maturation in the superficial layers. Characteristically, keratinocytes in the basal layers show abundant eosinophilic cytoplasm with prominent eosinophilic nucleoli and pronounced intercellular bridges (figure 2C). Dyskeratotic cells (parabasal cells showing premature maturation with eosinophilia and keration formation) are usually present throughout the epithelium (figure 2D). ${ }^{29}$ Keratinocytes in the mid to superficial layers may show focal or no atypia at all. Changes consistent with LS or lichen simplex chronicus can be found adjacent to dVIN or elsewhere in the resection specimen.

Recently van den Einden et al, ${ }^{29}$ demonstrated that agreement between pathologists in the diagnosis of dVIN is low and diagnosing dVIN is extremely difficult. According to a panel of pathologists, consensus was reached with characteristic histological features for the diagnosis of dVIN. Five histological criteria proved to be the most useful in the diagnosis of $\mathrm{dVIN}$ : atypical mitosis in the basal layer, basal cell atypia, dyskeratosis, prominent nucleoli, and elongation and anastomosis of the rete ridges. Following refinement of strict criteria, agreement with experienced gynaecological pathologists reached $\kappa$ values. Therefore, if high suspicion for dVIN is suspected clinically, the 
Figure 2 Vulvar intraepithelial neoplasia differentiated (simplex)-type dVIN. (A) Thickened parakeratotic epithelium with elongation and anastomosis of the rete ridges. (B) Atypical basal mitotic figures. (C) Basal cell cellular atypia with prominent nucleoli and intercellular bridges. (D) Dyskeratotic cells. (E) p53

immunohistochemistry, with positive cells extending above the basal layer.
A

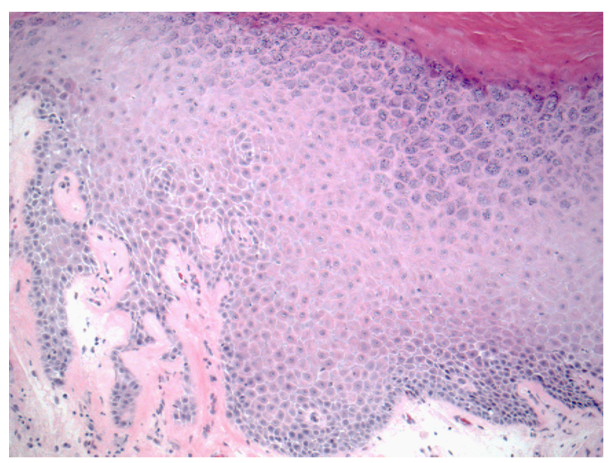

C

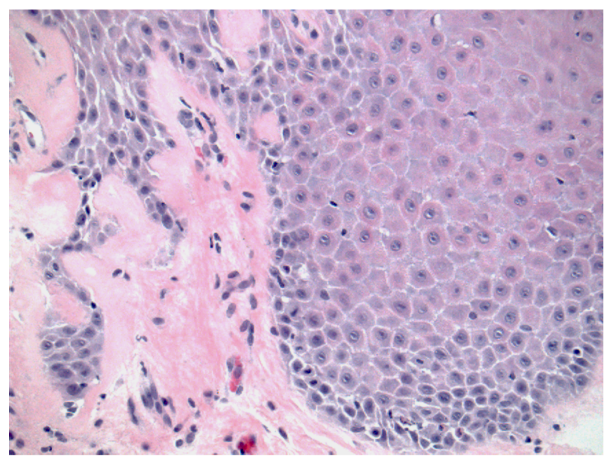

B

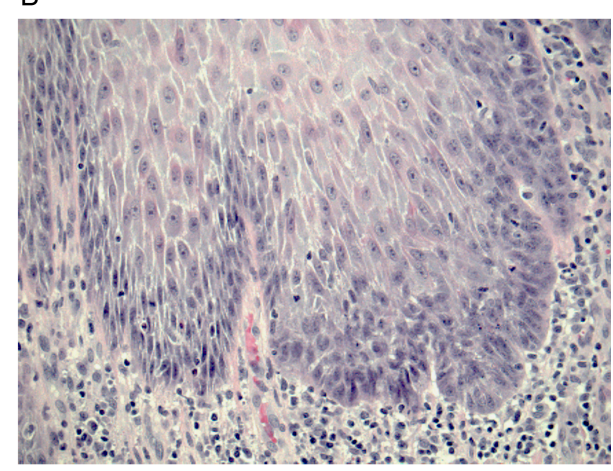

D

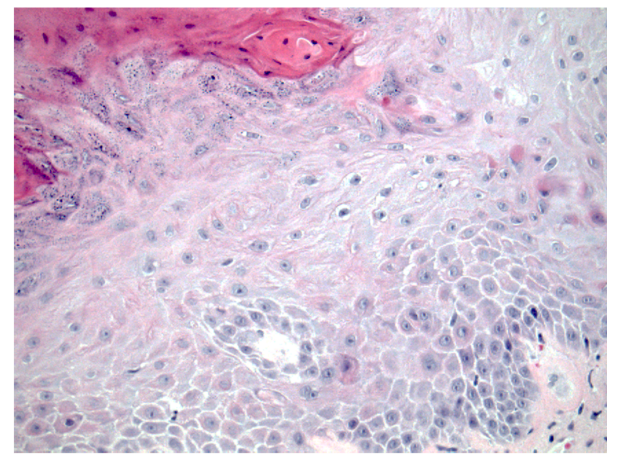

E

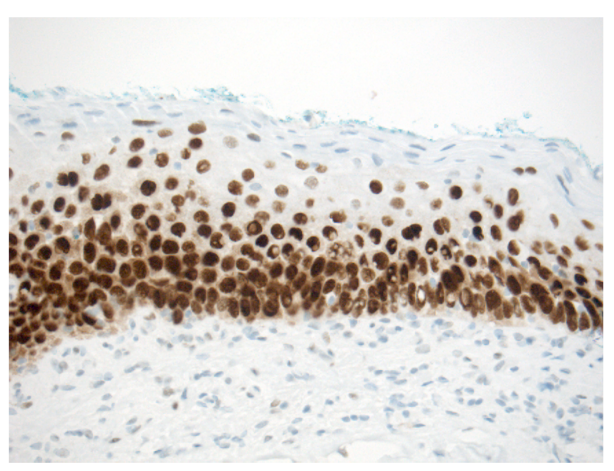

case should be reviewed by an experienced gynaecological pathologist.

By immunohistochemistry, basal cells stain positive for p53 with extension above the basal layer in $85-90 \%$ of dVIN (figure 2E). ${ }^{24}$ However, not all dVIN cases show TP53 mutations and therefore those cases will be negative for this marker. ${ }^{26}$ In addition, p53 immunostain has been shown to be positive in $25 \%$ normal vulvar epithelium cases and in up to $80 \%$ of LS cases. $^{27}{ }^{30}$ For these reasons, the use of p53 immunohistochemistry for the diagnosis of dVIN is limited with the refined morphological criteria being useful for diagnosis. In contrast to uVIN, p16 is not overexpressed.

\section{Differential diagnosis}

Differentiated VIN is characterized by basal nuclear atypia and this is the main feature that should be sought when considering a dVIN diagnosis. However, some cases may have mitotic figures and nuclear atypia that extend beyond the basal layer and in this setting uVIN may come in the differential diagnosis. In this instance the use of immunohistochemistry will help in the diagnosis. Negative or focally positive p16 staining would be consistent with dVIN. In addition, p53 immunostain can be performed with some dVIN cases showing p53 labelling above the basal layer. A comparison of differentiating clinicopathological features for uVIN and dVIN is shown in table 1.

Benign processes that show acanthosis and focal nuclear atypia also come in the differential diagnosis of dVIN. ${ }^{23}$ Pseudoepitheliomatous hyperplasia, a benign proliferative lesion of the squamous epithelium, may have dyskeratotic cells and pleomorphic nuclei. However, atypical basal mitotic figures and p53 labelling are not present in pseudoepitheliomatous hyperplasia. Inflammatory dermatological lesions such as LS and squamous hyperplasia can have focal atypia and even LS cases can express p53 by immunohistochemistry. Although LS can have basal cell proliferation and hyperchromasia, there won't be any nuclear pleomorphism.

\section{Natural history}

dVIN has a higher risk of progression to invasive SCC than uVIN (5.7\% vs 33\%, respectively). ${ }^{31-33}$ In addition the time of progression to SCC is significantly shorter in dVIN cases when compared with uVIN. ${ }^{33}$ 
Table 1 Comparison of clinicopathological features between uVIN and dVIN

\begin{tabular}{|c|c|c|}
\hline & uVIN & dVIN \\
\hline Prevalence & More common & Less common \\
\hline Age & Young women (40-49 years) & Postmenopausal women (66-69 years) \\
\hline Distribution & Multifocal & Usually unifocal \\
\hline Risk factors & HPV infection, immunosuppression & $\begin{array}{l}\text { Chronic skin inflammatory conditions (LS, lichen simplex chronicus, } \\
\text { squamous cell hyperplasia) }\end{array}$ \\
\hline Morphology & $\begin{array}{l}\text { Nuclear atypia (high nuclear/cytoplasmic ratios, nuclear } \\
\text { enlargement, hyperchromasia) } \\
\text { - Decreased cellular maturation } \\
\text { - Increased mitotic activity above basal layer }\end{array}$ & $\begin{array}{l}\text { Basal cell nuclear atypia } \\
\text { Atypical mitosis in basal layer } \\
\text { High maturation of superficial squamous cells } \\
\text { Dyskeratosis, prominent nucleoli, elongation and anastomosis of } \\
\text { rete ridges } \\
\text { Prominent intercellular bridges }\end{array}$ \\
\hline Type of SCC & Basaloid/warty SCC & Keratinizing SCC \\
\hline $\begin{array}{l}\text { Frequency of progression to } \\
\text { invasive cancer }\end{array}$ & $5.7 \%$ & $33 \%$ \\
\hline Immunohistochemistry & p16+ (diffuse, band-like), p53- & p53+(85\%), p16- or focally + \\
\hline
\end{tabular}

\section{Take home messages}

- There are two types of vulvar intraepithelial neoplasia (VIN): usual VIN and differentiated or simplex-type VIN (dVIN)

- UVIN is driven by human papilloma virus (HPV) infection, occurs in young women, is characterized by hyperchromatic nuclei, increased mitotic figures, increased nuclear to cytoplasmic ratios and overexpresses p16.

- dVIN is not related to viral infection, occurs in older women with chronic skin conditions, histological findings are subtle, they do not overexpress $\mathrm{p} 16$ and some are p53 positive.

Contributors $K C$ and MCR are the only contributors for this paper. MCR did the literature search and wrote the paper. KC reviewed and edited the paper, and made changes to it.

\section{Competing interests None.}

Provenance and peer review Not commissioned; internally peer reviewed.

\section{REFERENCES}

1 Judson PL, Habermann EB, Baxter NN, et al. Trends in the incidence of invasive and in situ vulvar carcinoma. Obstet Gynecol 2006;107:1018-22.

2 Del Pino M, Rodriguez-Carunchio L, Ordi J. Pathways of vulvar intraepithelial neoplasia and squamous cell carcinoma. Histopathology 2013;62:161-75.

3 Fox $\mathrm{H}$, Wells M. Recent advances in the pathology of the vulva. Histopathology 2003;42:209-16

4 Hart WR. Vulvar intraepithelial neoplasia: historical aspects and current status. Int $J$ Gynecol Pathol 2001;20:16-30.

5 McCluggage WG. Recent developments in vulvovaginal pathology. Histopathology 2009:54:156-73.

6 Sideri M, Jones RW, Wilkinson EJ, et al. Squamous vulvar intraepithelial neoplasia: 2004 modified terminology, ISSVD Vulvar Oncology Subcommittee. J Reprod Med 2005;50:807-10.

7 De Vuyst $\mathrm{H}$, Clifford GM, Nascimento MC, et al. Prevalence and type distribution of human papillomavirus in carcinoma and intraepithelial neoplasia of the vulva, vagina and anus: a meta-analysis. Int I Cancer 2009;124:1626-36.

8 Scurry J, Campion M, Scurry B, et al. Pathologic audit of 164 consecutive cases of vulvar intraepithelial neoplasia. Int I Gynecol Pathol 2006;25:176-81.

9 de Bie RP, van de Nieuwenhof HP, Bekkers RL, et al. Patients with usual vulvar intraepithelial neoplasia-related vulvar cancer have an increased risk of cervical abnormalities. Br J Cancer 2009:101:27-31.

10 Sykes $\mathrm{P}$, Smith N, McCormick P, et al. High-grade vulval intraepithelial neoplasia (VIN 3): a retrospective analysis of patient characteristics, management, outcome and relationship to squamous cell carcinoma of the vulva 1989-1999. Aust N Z J Obstet Gynaecol 2002;42:69-74.

11 Joura EA, Losch A, Haider-Angeler MG, et al. Trends in vulvar neoplasia. Increasing incidence of vulvar intraepithelial neoplasia and squamous cell carcinoma of the vulva in young women. J Reprod Med 2000;45:613-15.

12 Feng Q, Kiviat NB. New and surprising insights into pathogenesis of multicentric squamous cancers in the female lower genital tract. J Natl Cancer Inst 2005:97:1798-9.

13 Logani S, Lu D, Quint WG, et al. Low-grade vulvar and vaginal intraepithelial neoplasia: correlation of histologic features with human papillomavirus DNA detection and MIB-1 immunostaining. Mod Pathol 2003;16:735-41.

14 Srodon M, Stoler MH, Baber GB, et al. The distribution of low and high-risk HPV types in vulvar and vaginal intraepithelial neoplasia (VIN and VaIN). Am I Surg Pathol 2006;30:1513-18.

15 Heller DS. Report of a new ISSVD classification of VIN. J Low Genit Tract Dis 2007;11:46-7.

16 Rufforny I, Wilkinson EJ, Liu C, et al. Human papillomavirus infection and p16 (INK4a) protein expression in vulvar intraepithelial neoplasia and invasive squamous cell carcinoma. J Lower Genit Tract Dis 2005;9:108-13.

17 McCluggage WG. Recent advances in immunohistochemistry in gynaecological pathology. Histopathology 2002;40:309-26.

18 McCluggage WG, Jamison J, Boyde A, et al. Vulval intraepithelial neoplasia with mucinous differentiation: report of 2 cases of a hitherto undescribed phenomenon. Am J Surg Pathol 2009;33:945-9.

19 van Seters M, van Beurden M, de Craen AJ. Is the assumed natural history of vulvar intraepithelial neoplasia III based on enough evidence? A systematic review of 3322 published patients. Gynecol Oncol 2005;97:645-51.

20 Jones RW. The natural history of cervical and vulvar intraepithelial neoplasia. Am J Obstet Gynecol 2010;202:e12-13.

21 Jones RW, Rowan DM, Stewart AW. Vulvar intraepithelial neoplasia: aspects of the natural history and outcome in 405 women. Obstet Gynecol 2005;106:1319-26.

22 Jones RW, Rowan DM. Spontaneous regression of vulvar intraepithelial neoplasia 2-3. Obstet Gynecol 2000;96:470-2.

23 Mulvany NJ, Allen DG. Differentiated intraepithelial neoplasia of the vulva. Int J Gynecol Pathol 2008;27:125-35.

24 Yang B, Hart WR. Vulvar intraepithelial neoplasia of the simplex (differentiated) type: a clinicopathologic study including analysis of HPV and p53 expression. Am J Surg Pathol 2000;24:429-41.

25 Carlson JA, Ambros R, Malfetano J, et al. Vulvar lichen sclerosus and squamous cell carcinoma: a cohort, case control, and investigational study with historical perspective; implications for chronic inflammation and sclerosis in the development of neoplasia. Hum Pathol 1998;29:932-48.

26 Pinto AP, Miron A, Yassin Y, et al. Differentiated vulvar intraepithelial neoplasia contains Tp53 mutations and is genetically linked to vulvar squamous cell carcinoma. Mod Pathol 2010:23:404-12.

27 Rolfe KJ, MacLean AB, Crow JC, et al. TP53 mutations in vulval lichen sclerosus adjacent to squamous cell carcinoma of the vulva. Br J Cancer 2003;89:2249-53.

28 McNally OM, Mulvany NJ, Pagano R, et al. VIN 3: a clinicopathologic review. Int J Gynecol Cancer 2002;12:490-5.

29 van den Einden LC, de Hullu JA, Massuger LF, et al. Interobserver variability and the effect of education in the histopathological diagnosis of differentiated vulvar intraepithelial neoplasia. Mod Pathol 2013;26:874-80. 


\section{Review}

30 Liegl B, Regauer S. p53 immunostaining in lichen sclerosus is related to ischaemic stress and is not a marker of differentiated vulvar intraepithelial neoplasia (d-VIN). Histopathology 2006;48:268-74.

31 Eva LJ, Ganesan R, Chan KK, et al. Differentiated-type vulval intraepithelial neoplasia has a high-risk association with vulval squamous cell carcinoma. Int J Gynecol Cancer 2009;19:741-4.
32 Eva LJ, Ganesan R, Chan KK, et al. Vulval squamous cell carcinoma occurring on a background of differentiated vulval intraepithelial neoplasia is more likely to recur: a review of 154 cases. J Reprod Med 2008;53:397-401.

33 van de Nieuwenhof HP, van Kempen LC, Massuger LF, et al. "Differentiated-type vulval intraepithelial neoplasia has a high-risk association with vulval squamous cell carcinoma". Int J Gynecol Cancer 2010;20:194. 